\title{
Revised mechanisms of the catalytic alcohol dehydrogenation and ester reduction with the Milstein PNN complex of ruthenium
}

\author{
Dmitry G. Gusev* \\ Department of Chemistry and Biochemistry, Wilfrid Laurier University, Waterloo ON N2L 3C5 \\ Canada
}

E-mail: dgoussev@wlu.ca

Table of Contents

Representative pictures of the crystalline $2 \quad$ p. S2

NMR spectra

p. S3 - S13

Computed energies

p. $\$ 14-16$

Crystal Data Collection and Refinement Parameters for 2-Os

p. $517-23$ 

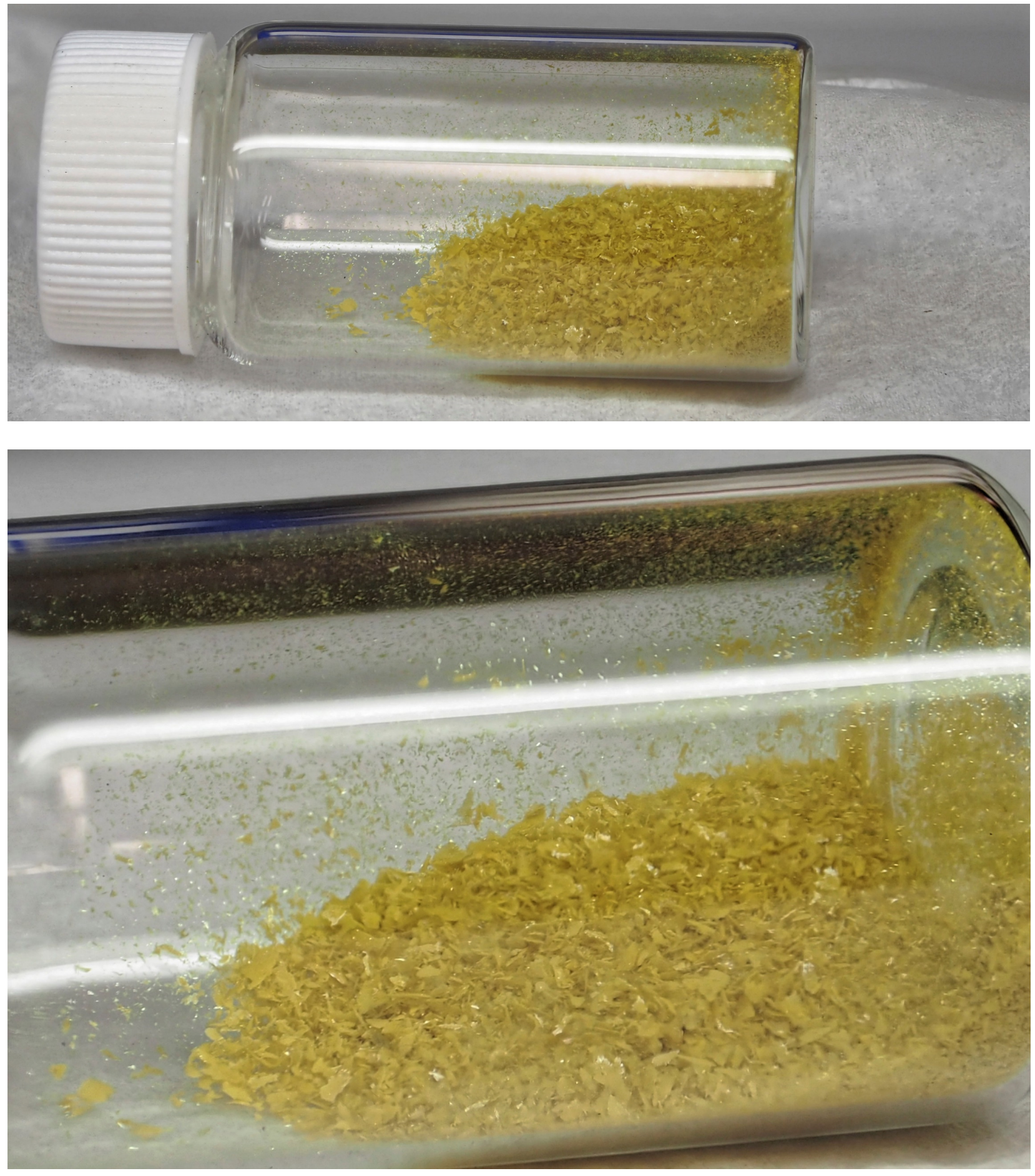

Figure S1. Complex $2(0.37 \mathrm{~g})$ in a $20 \mathrm{~mL}$ vial, under Ar. 


\section{${ }^{1} \mathrm{H}(400 \mathrm{MHz})$ and ${ }^{13} \mathrm{C}$ NMR $(100 \mathrm{MHz})$ spectra}
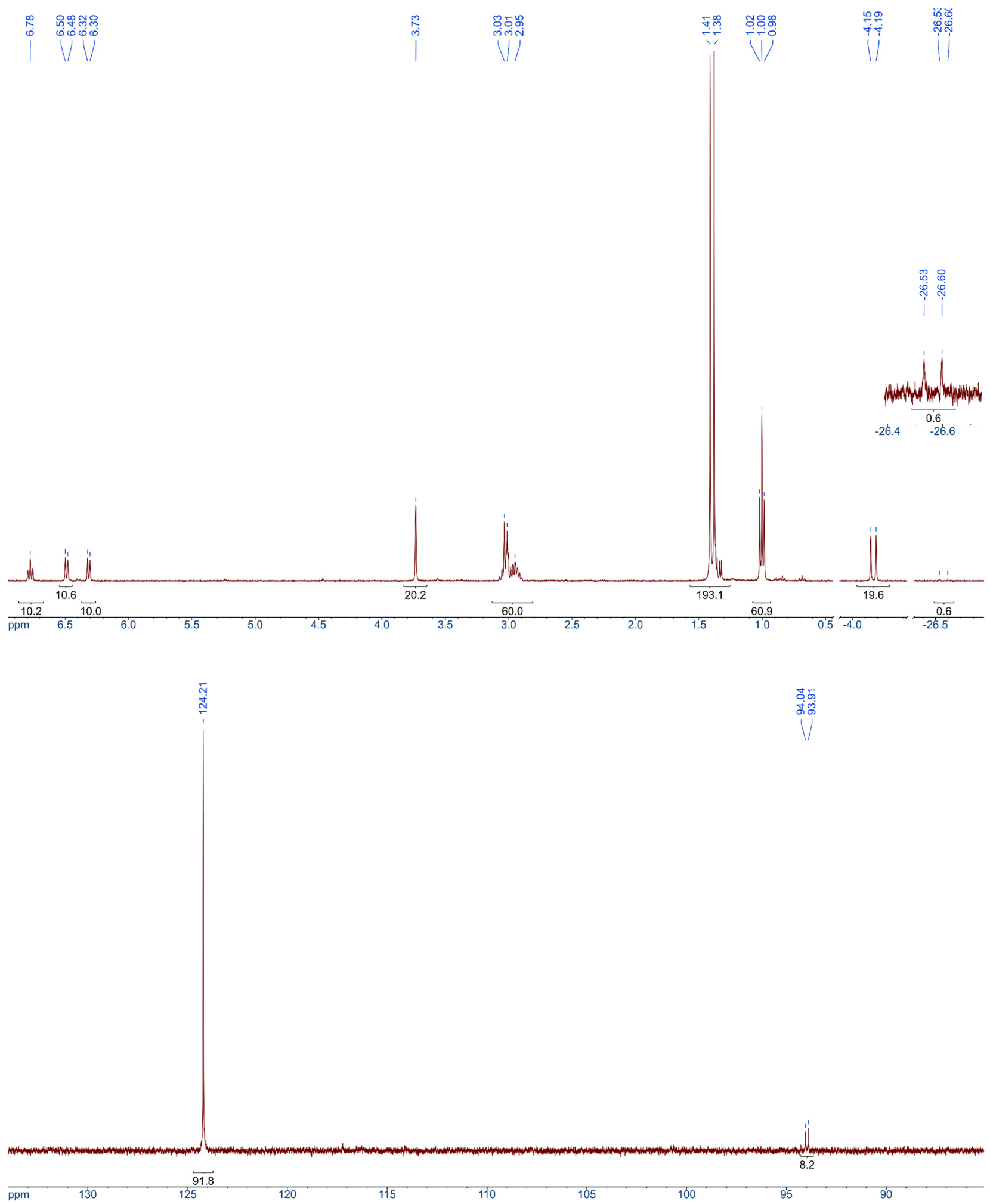

Figure S2. ${ }^{31} \mathrm{P}\left\{{ }^{1} \mathrm{H}\right\}$ and ${ }^{1} \mathrm{H}$ NMR spectra of a saturated solution of $2\left(2.4 \times 10^{-6} \mathrm{~mol}\right)$ in $\mathrm{C}_{6} \mathrm{D}_{6}$ (ca. $\left.0.0074 \mathrm{M}\right)$. The ${ }^{31} \mathrm{P}$ NMR resonance of 1 at $93.98 \mathrm{ppm}$ shows coupling to the hydride, because the chemicals shift of $\mathrm{RuH}(\delta-26.57)$ falls outside the decoupler window. 


\section{Supporting Information}
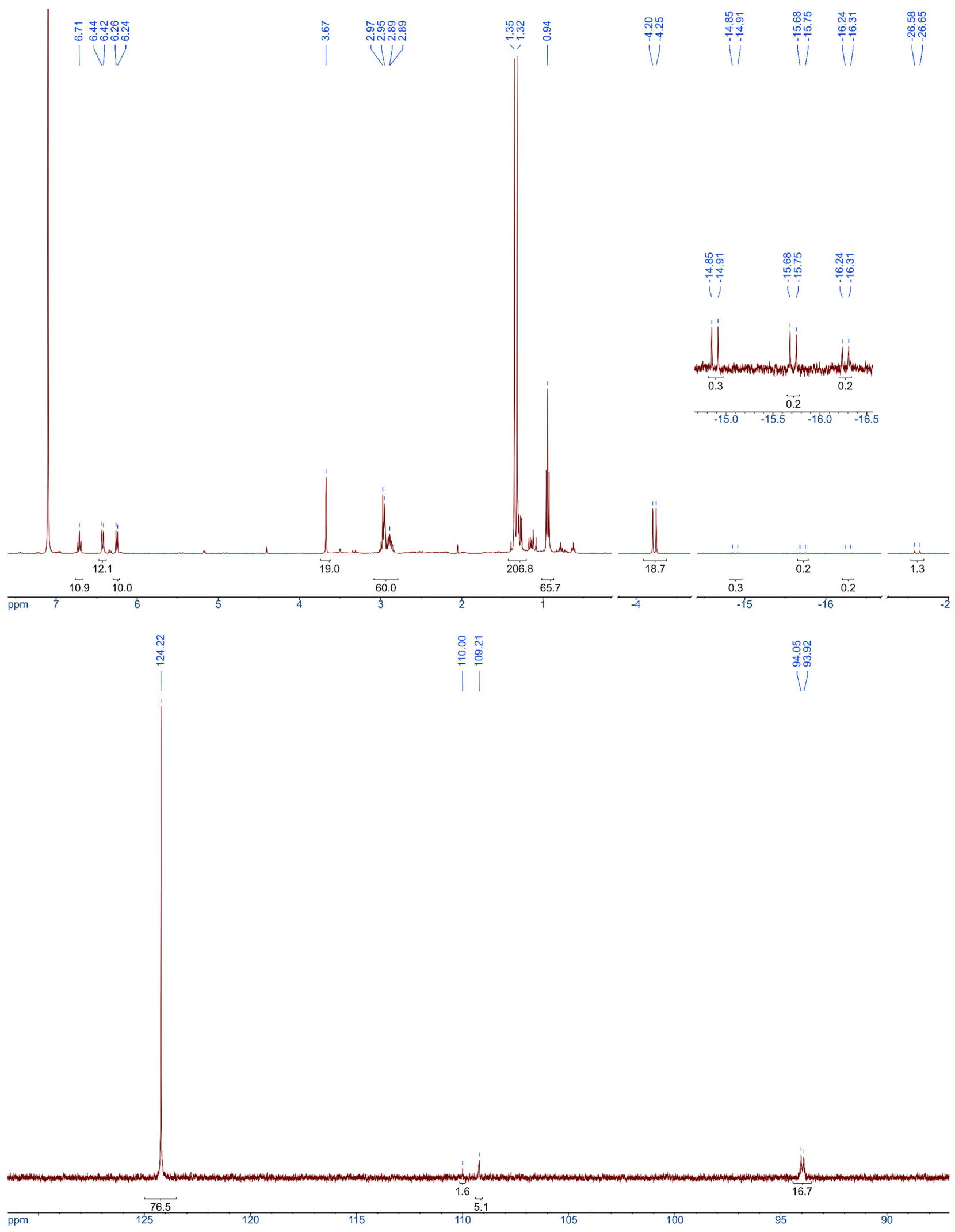

Figure S3. ${ }^{31} \mathrm{P}\left\{{ }^{1} \mathrm{H}\right\}$ and ${ }^{1} \mathrm{H}$ NMR spectra $\left(\mathrm{C}_{6} \mathrm{D}_{6}\right.$ solvent) of a sample of 2 left in air overnight. The ${ }^{31} \mathrm{P}$ NMR resonance of 1 at $93.98 \mathrm{ppm}$ shows coupling to the hydride, because the chemicals shift of $\mathrm{RuH}(\delta-26.57)$ falls outside the decoupler window. 

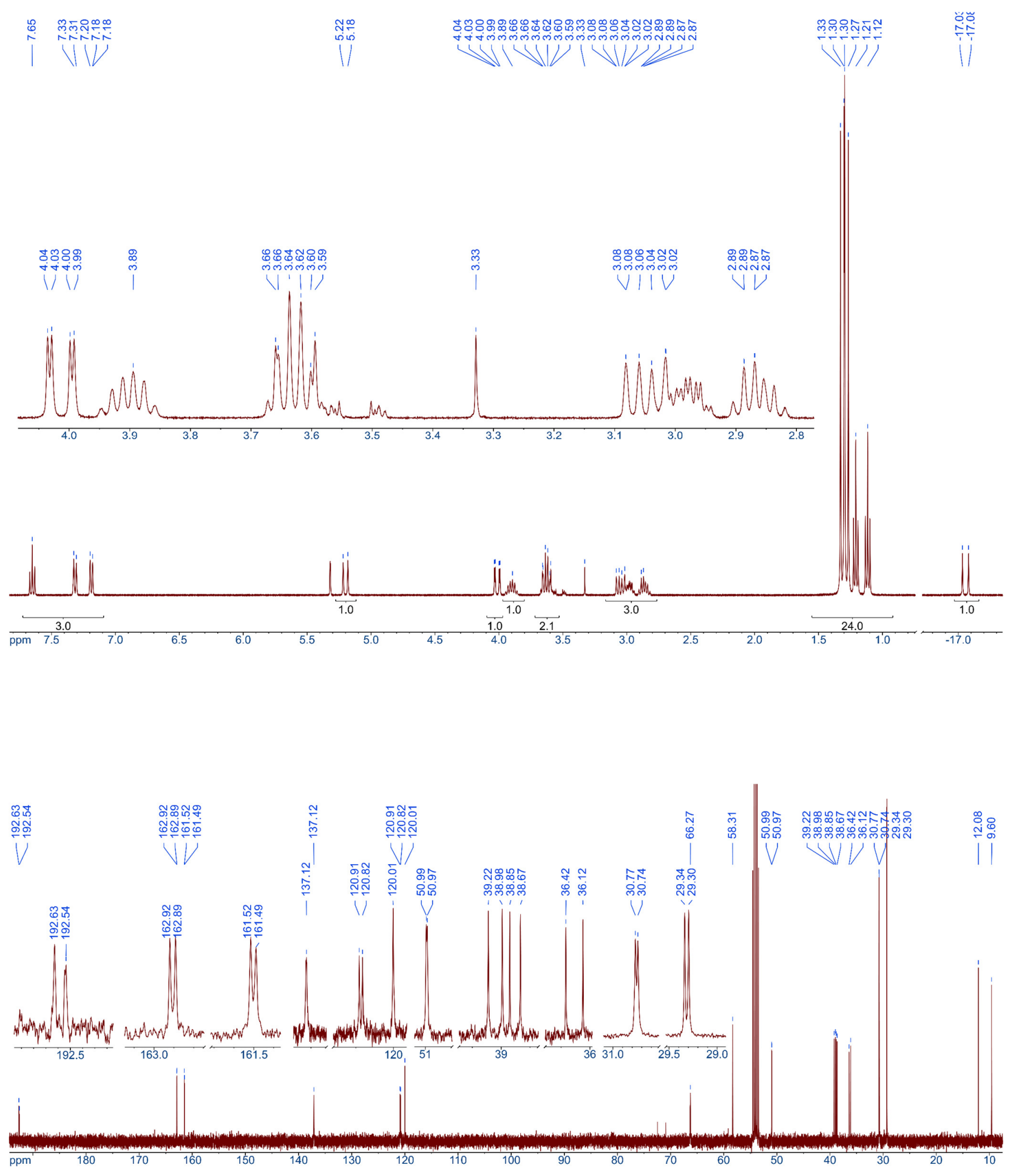

Figure S4. ${ }^{13} \mathrm{C}\left\{{ }^{1} \mathrm{H}\right\}$ and ${ }^{1} \mathrm{H}$ NMR spectra of $\mathrm{OsHCl}(\mathrm{CO})\left[\mathrm{Et}_{2} \mathrm{NCH}_{2} \mathrm{PyCH}_{2} \mathrm{PtBu}{ }_{2}\right]$ in $\mathrm{CD}_{2} \mathrm{Cl}_{2}$ 


\section{Supporting Information}

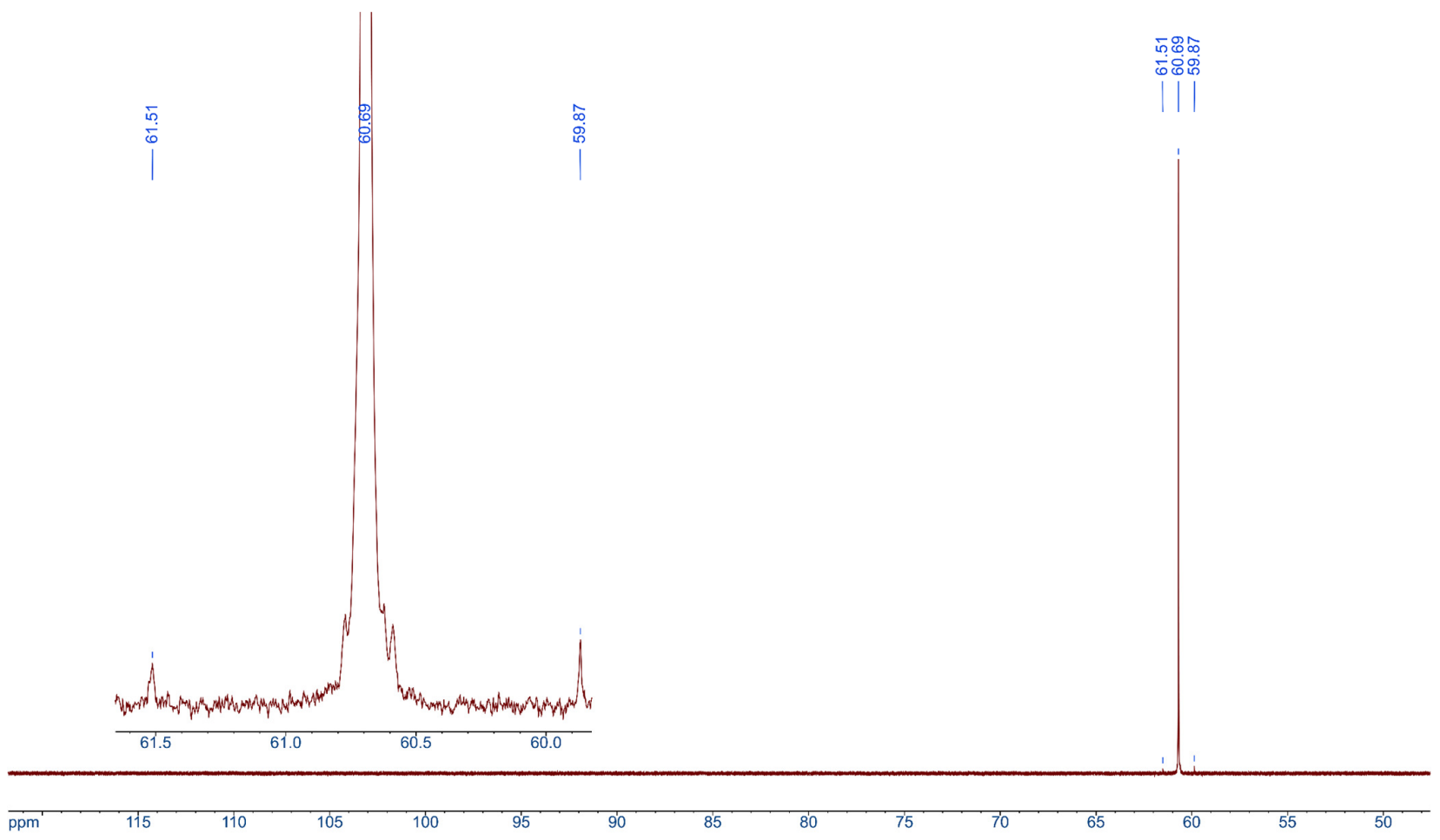

Figure S5. ${ }^{31} \mathrm{P}\left\{{ }^{1} \mathrm{H}\right\} \mathrm{NMR}$ spectrum of $\mathrm{OsHCl}(\mathrm{CO})\left[\mathrm{Et}_{2} \mathrm{NCH}_{2} \mathrm{PyCH}_{2} \mathrm{PtBu} 2\right]$ in $\mathrm{CD}_{2} \mathrm{Cl}_{2}$.

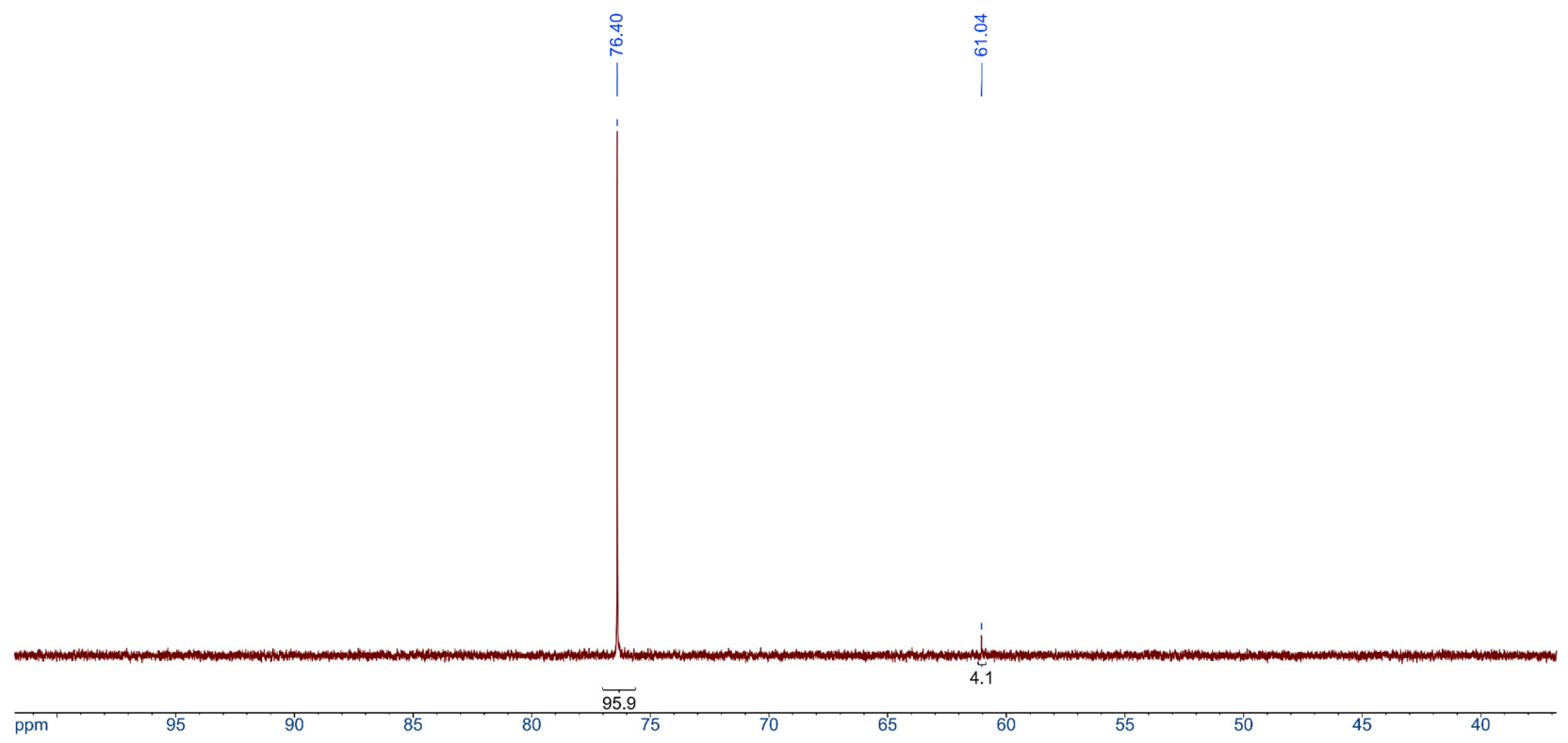

Figure S6. ${ }^{31} \mathrm{P}\left\{{ }^{1} \mathrm{H}\right\}$ NMR spectrum of $2-\mathrm{O}$ s in $\mathrm{C}_{6} \mathrm{D}_{6}$. 

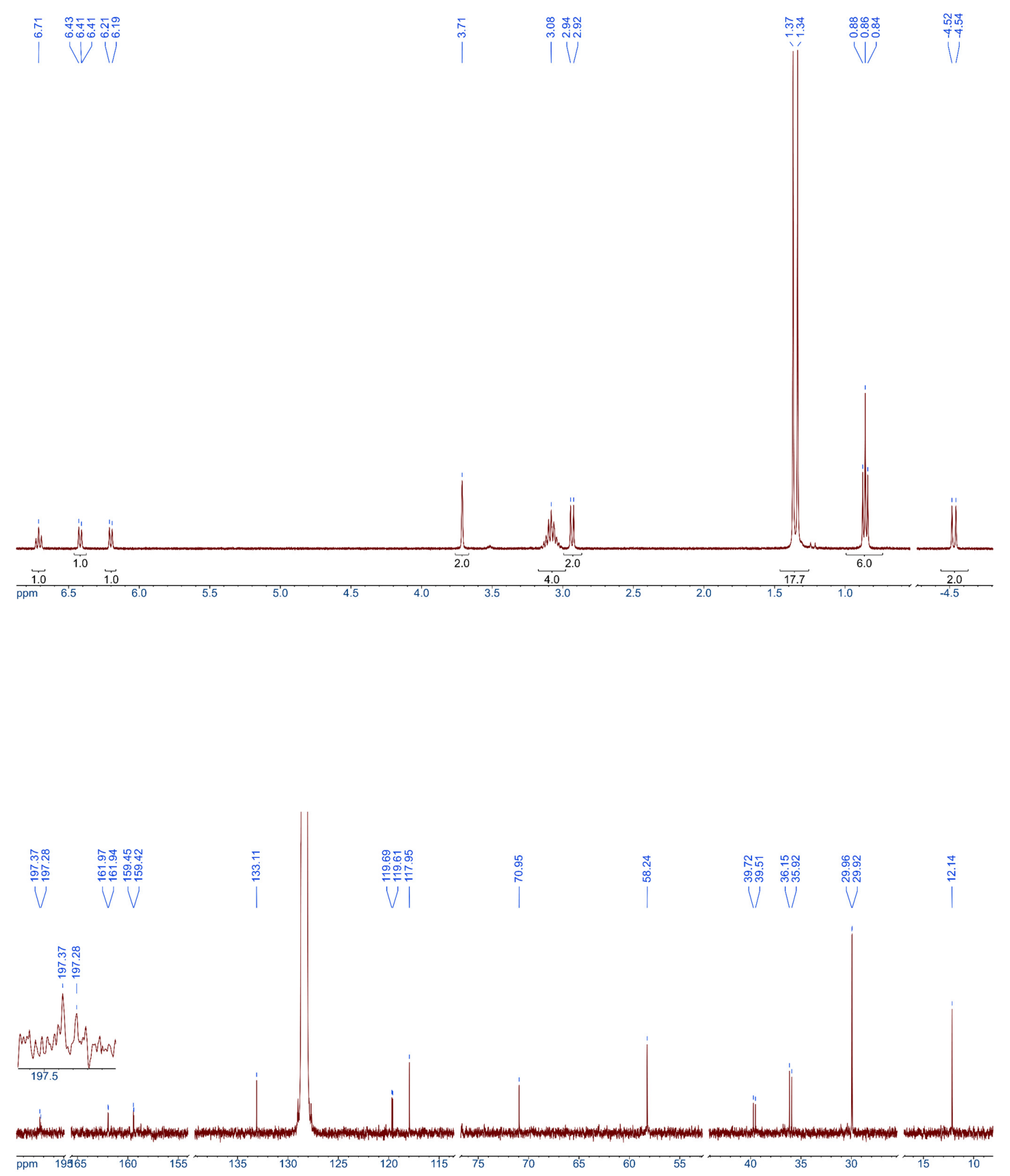

Figure S7. ${ }^{1} \mathrm{H}$ and ${ }^{13} \mathrm{C}\left\{{ }^{1} \mathrm{H}\right\}$ NMR spectra of $2-\mathrm{O}$ s in $\mathrm{C}_{6} \mathrm{D}_{6}$ 


\section{Supporting Information}

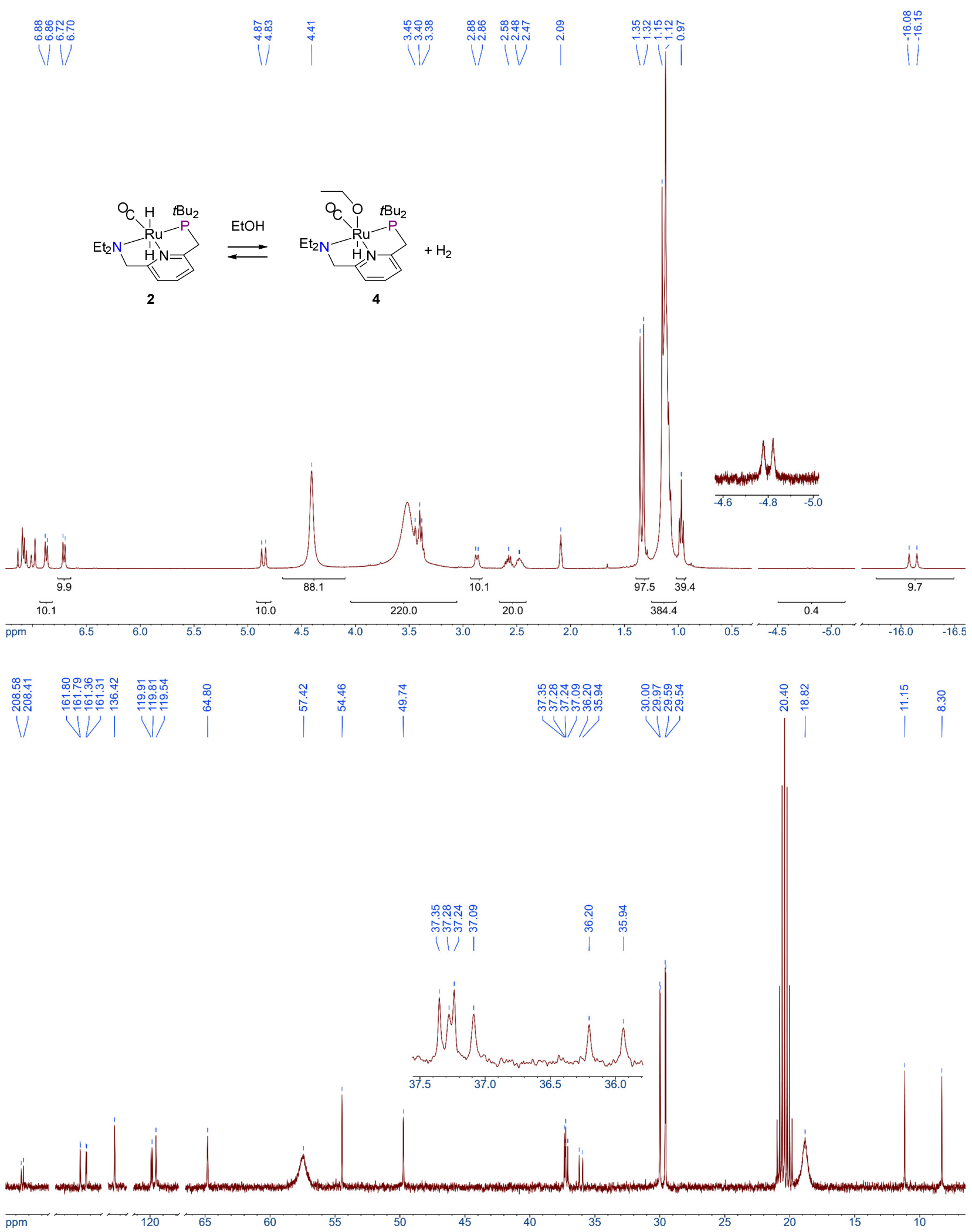

Figure S8. ${ }^{1} \mathrm{H}$ and ${ }^{13} \mathrm{C}\left\{{ }^{1} \mathrm{H}\right\}$ NMR spectra of $2\left(0.1 \mathrm{M}\right.$ solution in toluene- $\mathrm{d}_{8}$, prepared under $\left.\mathrm{Ar}\right)$ with 9.2 equiv of $\mathrm{EtOH}$, displaying the resonances of $\mathbf{4}$ as the principal metal species in solution (98\%). 


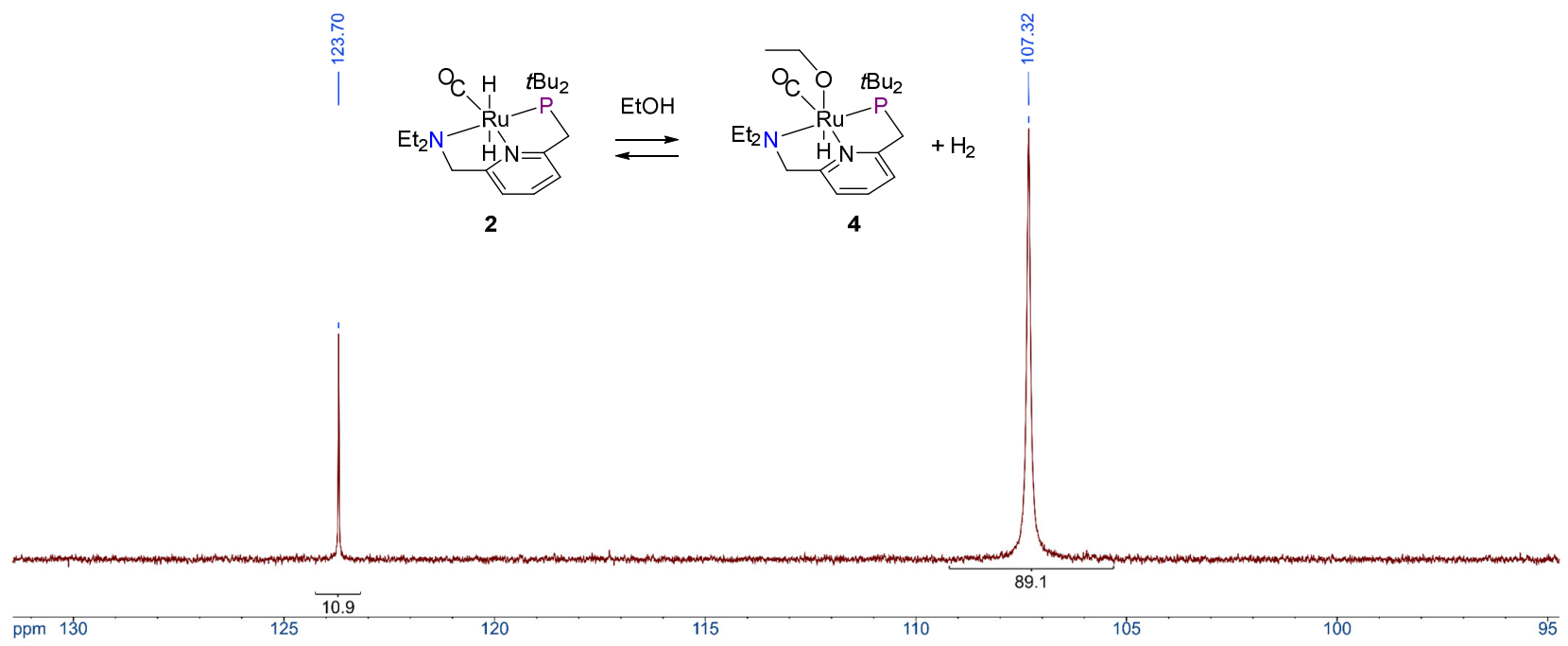

Figure S9. ${ }^{31} \mathrm{P}\left\{{ }^{1} \mathrm{H}\right\}$ NMR spectrum of $2(0.1 \mathrm{M}$ solution in toluene-d8, prepared under Ar) with 9.2 equiv of EtOH.

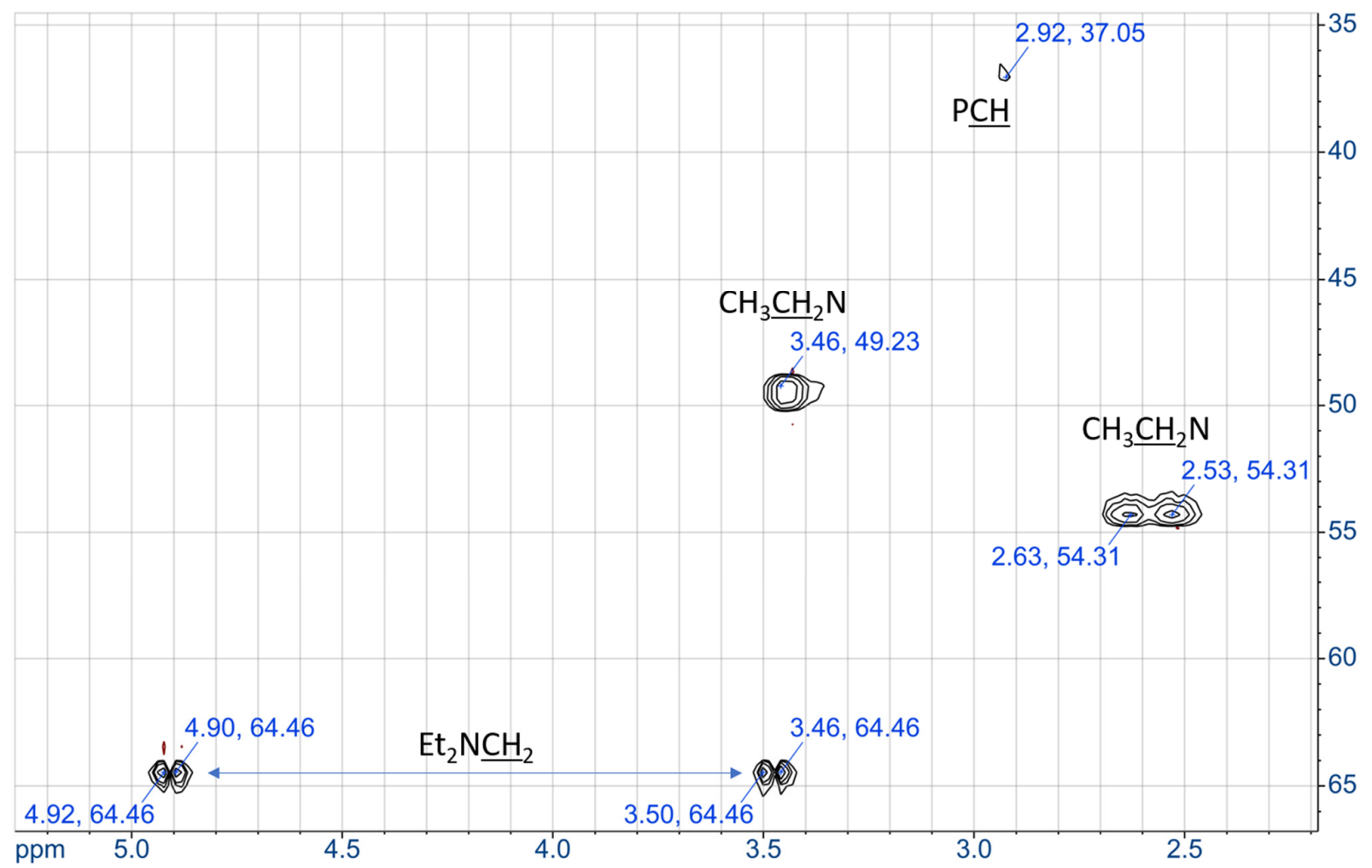

Figure S10. ${ }^{1} \mathrm{H} /{ }^{13} \mathrm{C}$ correlated HMQC $2 \mathrm{D}$ NMR spectrum of $2(0.1 \mathrm{M}$ solution in toluene-d8, prepared under $\mathrm{Ar})$ with 9.2 equiv of $\mathrm{EtOH}$, displaying the $\mathrm{CH}_{2}$ resonances of 4 (the principal species in solution). 


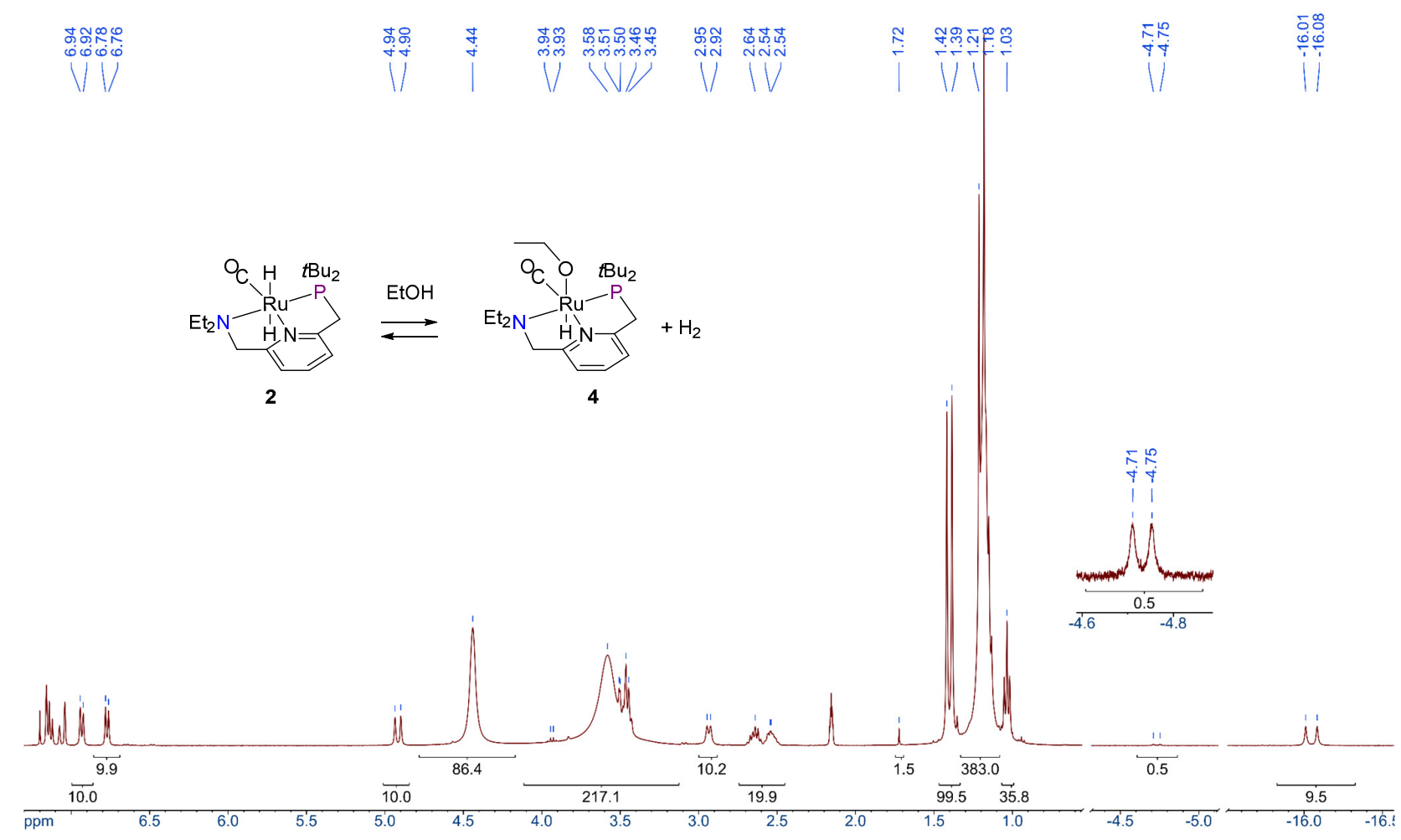

Figure S11. ${ }^{1} \mathrm{H}$ NMR spectrum of $2(0.1 \mathrm{M}$ solution in toluene-d8, prepared under Ar) with 9.2 equiv of EtOH, after 2 days at room temperature. 4 is the principal species in solution (98\%). See Figure S8 for comparison with the ${ }^{1} \mathrm{H}$ NMR spectrum of this solution immediately after preparation.

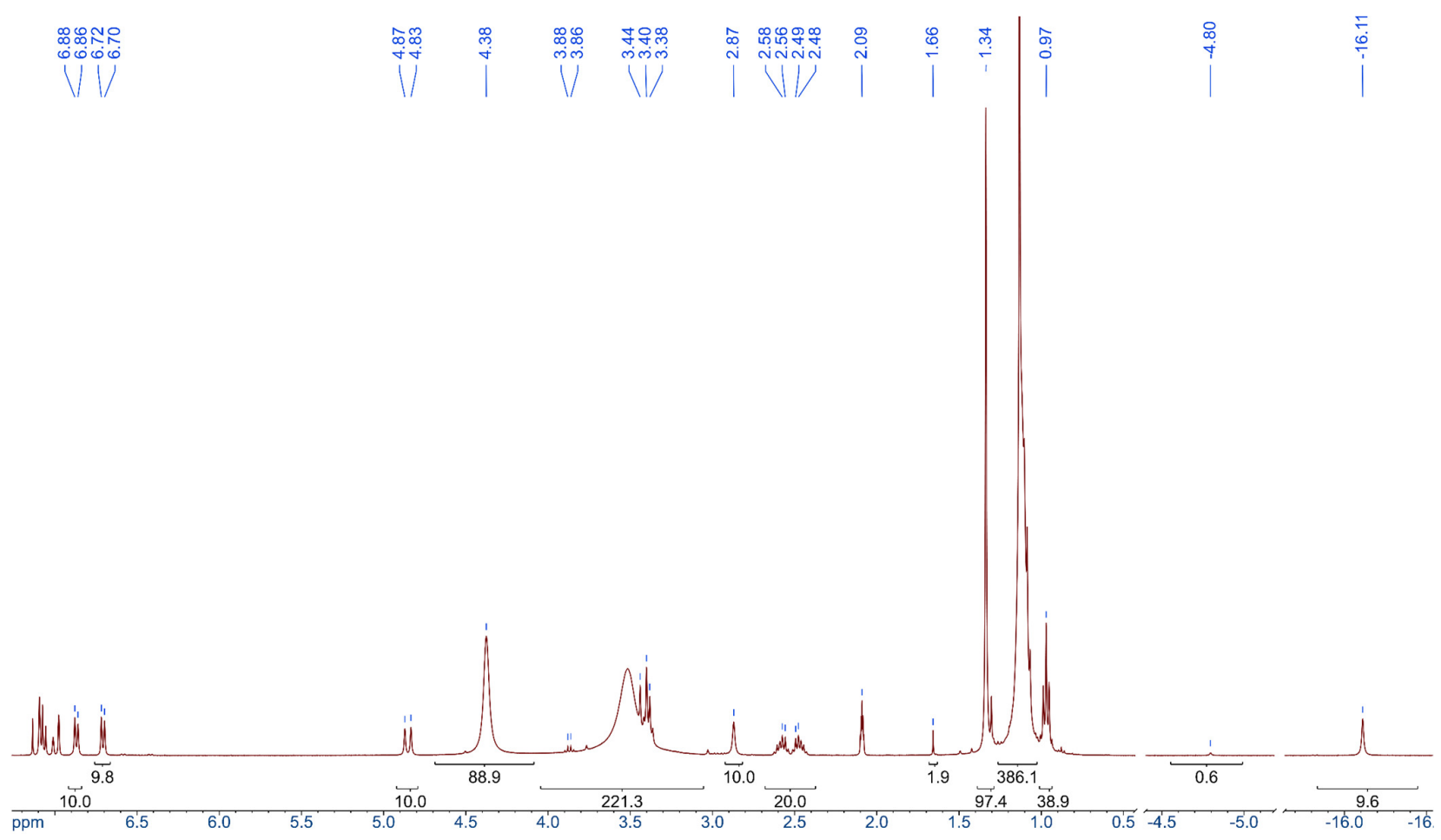

Figure S12. ${ }^{1} \mathrm{H}\left\{{ }^{31} \mathrm{P}\right\}$ NMR spectrum of $2(0.1 \mathrm{M}$ solution in toluene-d8, prepared under Ar) with 9.2 equiv of EtOH, after 2 days at room temperature. 4 is the principal species in solution (98\%). For comparison with the ${ }^{1} \mathrm{H}$ NMR spectrum of this solution see Figure S11. 


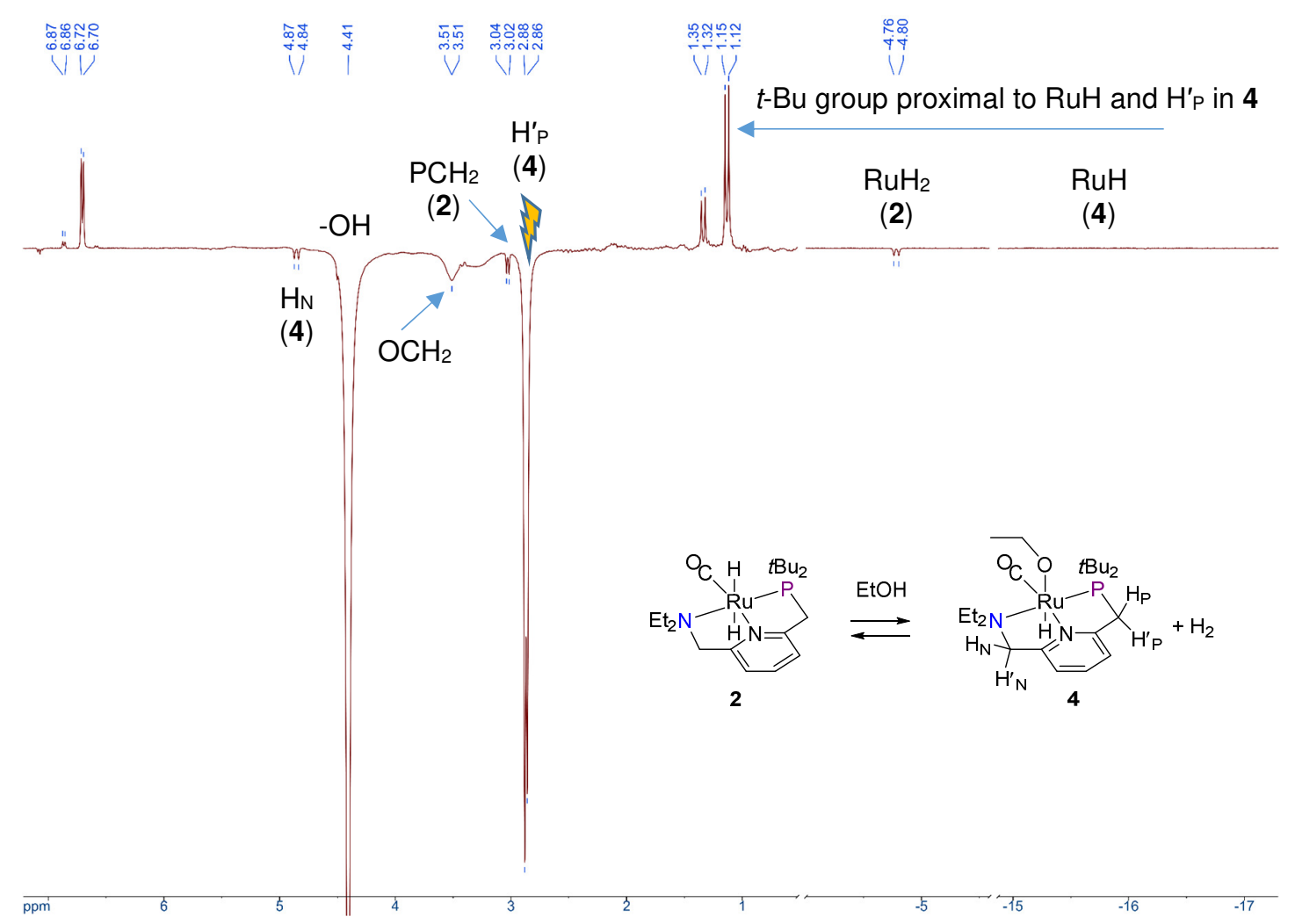

Figure S13. A difference NOE/saturation transfer ${ }^{1} \mathrm{H}$ NMR spectrum of $2(0.1 \mathrm{M}$ solution in toluene-d 8 , prepared under $\mathrm{Ar}$ ) with 9.2 equiv of $\mathrm{EtOH} .4$ is the principal species in solution (98\%). The $\mathrm{CH}$ resonance of 4 at $2.87 \mathrm{ppm}$ was irradiated.

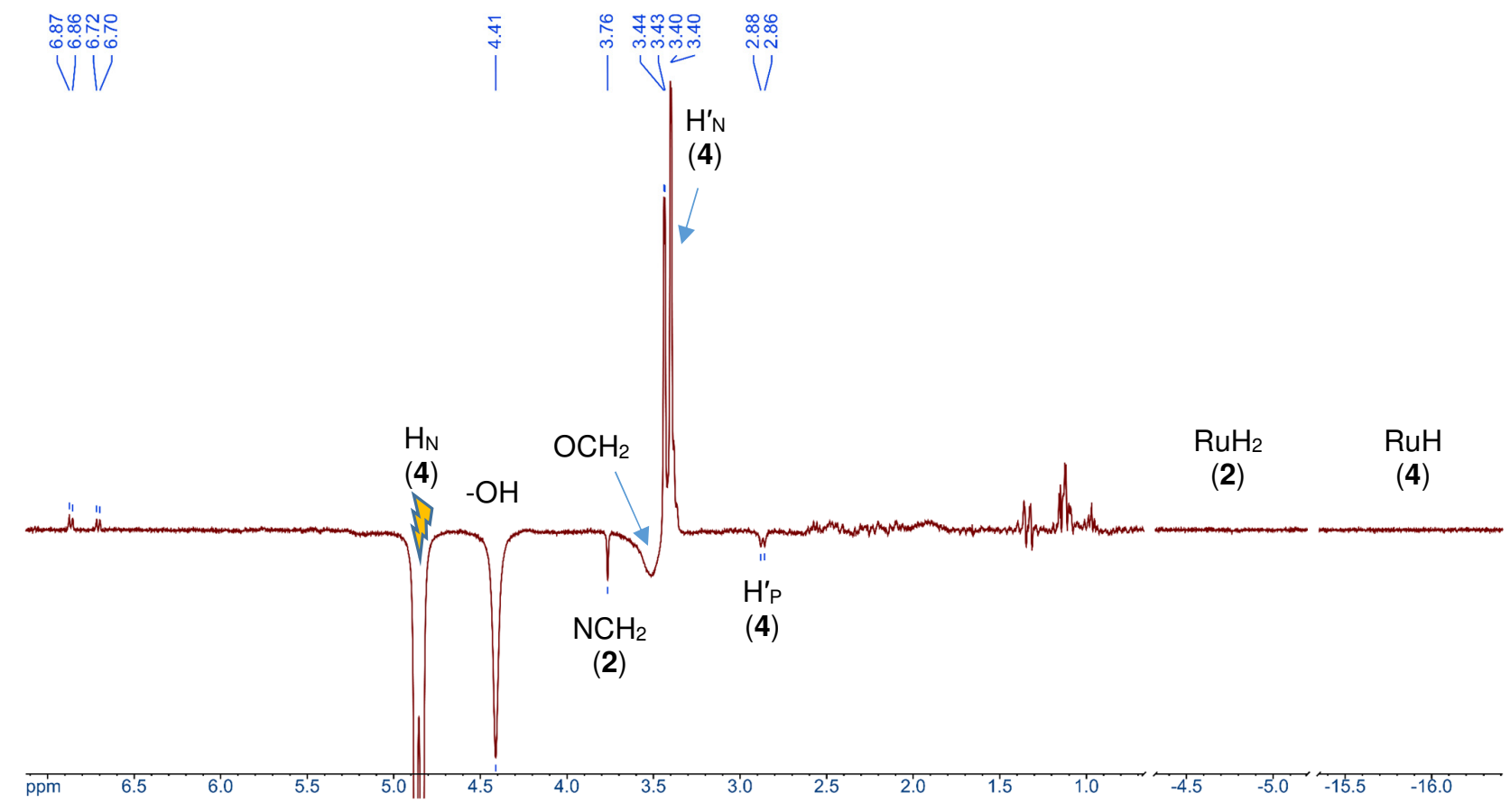

Figure S14. A difference NOE/saturation transfer ${ }^{1} \mathrm{H}$ NMR spectrum of $2\left(0.1 \mathrm{M}\right.$ solution in toluene- $\mathrm{d}_{8}$, prepared under $\mathrm{Ar}$ ) with 9.2 equiv of $\mathrm{EtOH} .4$ is the principal species in solution (98\%). The $\mathrm{CH}$ resonance of 4 at $4.86 \mathrm{ppm}$ was irradiated. 
Supporting Information

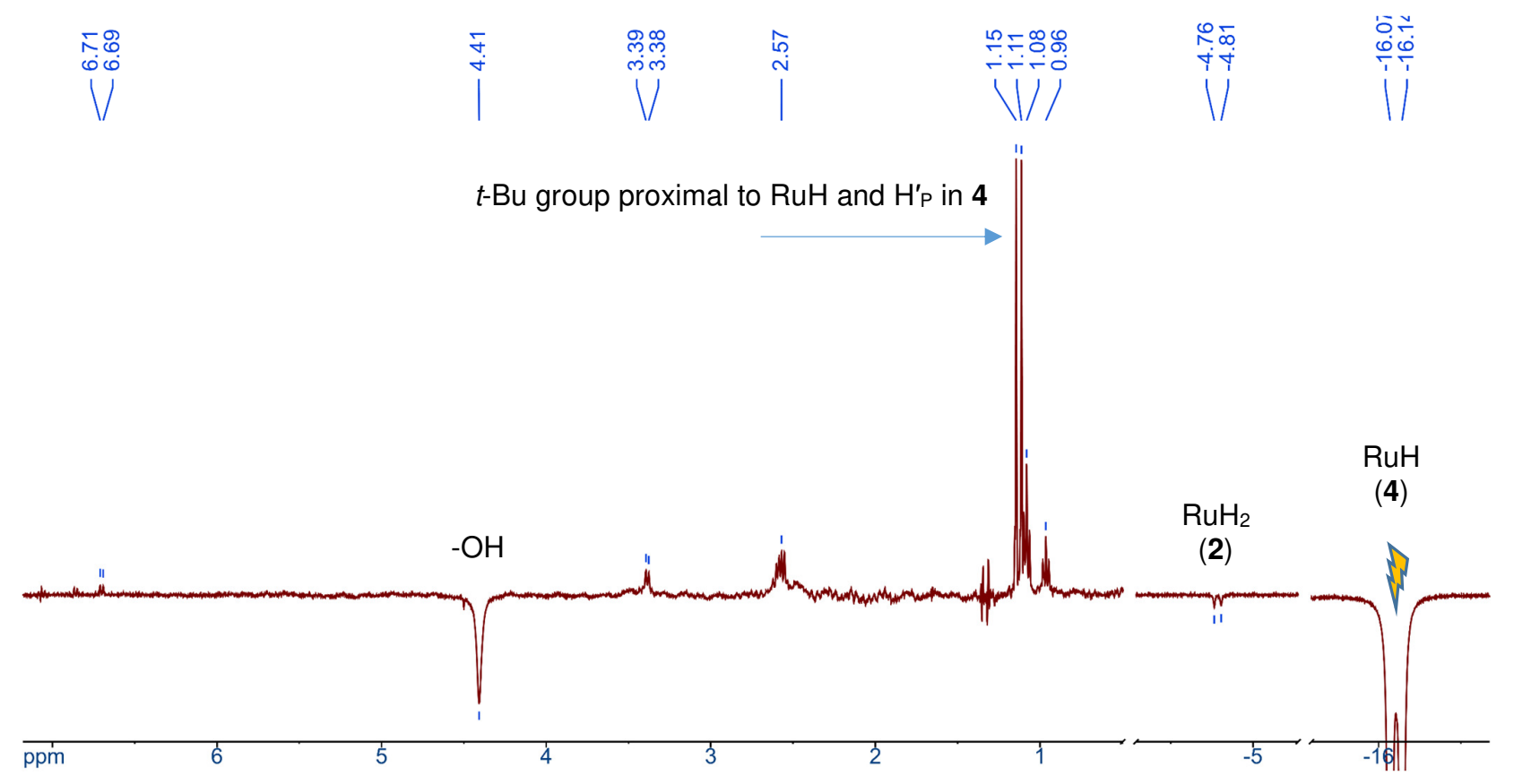

Figure S15. A difference NOE/saturation transfer ${ }^{1} \mathrm{H}$ NMR spectrum of $2\left(0.1 \mathrm{M}\right.$ solution in toluene- $\mathrm{d}_{8}$, prepared under $\mathrm{Ar}$ ) with 9.2 equiv of $\mathrm{EtOH} .4$ is the principal species in solution (98\%). The RuH resonance of 4 at -16.11 ppm was irradiated. 

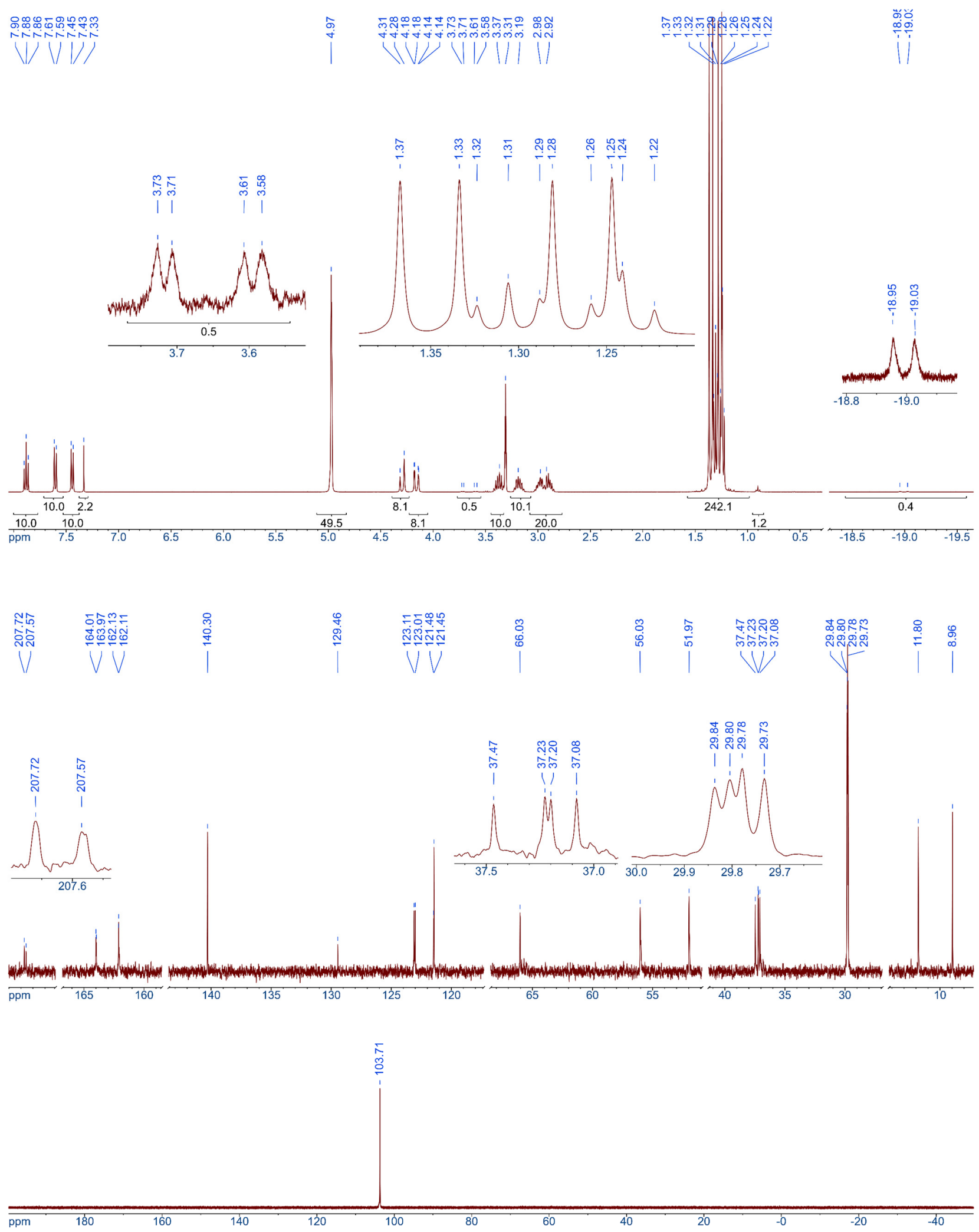

Figure S16. ${ }^{1} \mathrm{H},{ }^{13} \mathrm{C}\left\{{ }^{1} \mathrm{H}\right\}$ and ${ }^{31} \mathrm{P}\left\{{ }^{1} \mathrm{H}\right\}$ NMR spectra of 2 dissolved in $\mathrm{CD}_{3} \mathrm{OD}$, displaying formation of $\operatorname{RuD}\left(\mathrm{OCD}_{3}\right)(\mathrm{CO})\left[\mathrm{Et}_{2} \mathrm{NCH}_{2} \mathrm{PyCD}_{2} \mathrm{PtBu} \mathrm{Bu}_{2}\right]$. The ${ }^{1} \mathrm{H}$ NMR spectrum exhibits the resonances of $\mathrm{C}_{6} \mathrm{H}_{6}(\delta 7.33)$ and hexane co-crystalized with 2 during the preparation of the complex. 


\section{Supporting Information}

\section{Computed energies}

Table S1. M06-L energies (in toluene solvent continuum).

\begin{tabular}{|c|c|c|c|c|}
\hline species & Imag. Freq. & $E$ & $\mathrm{H}$ & $G$ \\
\hline $\mathrm{H}_{2}(1 \mathrm{~atm})$ & & -1.167333 & -1.154011 & -1.168832 \\
\hline Acetaldehyde & & -153.708957 & -153.648900 & -153.678648 \\
\hline $\mathrm{EtOH}$ & & -154.915165 & -154.830211 & -154.860791 \\
\hline EtOAc & & -307.462506 & -307.336462 & -307.377125 \\
\hline $\mathrm{H}_{2} \mathrm{O}$ & & -76.369128 & -76.343880 & -76.365306 \\
\hline 1-(ethoxy)ethanol & & -308.639622 & -308.490444 & -308.530913 \\
\hline 1 & & -1403.507604 & -1402.960213 & -1403.046270 \\
\hline $\mathbf{1} \cdot(\mathrm{EtOH})_{2}$ & & -1713.366587 & -1712.644949 & -1712.759016 \\
\hline $\mathbf{1}^{\prime} \cdot(\mathrm{EtOH})_{2}$ & & -1713.364406 & -1712.641962 & -1712.750478 \\
\hline 2 & & -1404.705671 & -1404.139094 & -1404.224580 \\
\hline TS1 & $i=-1777.52$ & -1404.659397 & -1404.098589 & -1404.184703 \\
\hline Int 8 & & -1404.688802 & -1404.123438 & -1404.210321 \\
\hline Complex 4 & & -1558.452875 & -1557.818341 & -1557.914036 \\
\hline $\mathrm{RuH}(\mathrm{OH})(\mathrm{CO})\left[\mathrm{Et}_{2} \mathrm{NCH}_{2} \mathrm{PyCH}_{2} \mathrm{PtBu}_{2}\right]$ & & -1479.901899 & -1479.326258 & -1479.415070 \\
\hline $\begin{array}{l}\text { 1-(ethoxy)ethoxide, } \\
\mathrm{RuH}\left\{\mathrm{OCH}(\mathrm{OEt}) \mathrm{CH}_{3}\right\}(\mathrm{CO})\left[\mathrm{Et}_{2} \mathrm{NCH}_{2} \mathrm{PyCH}_{2} \mathrm{PtBu}_{2}\right]\end{array}$ & & -1712.178090 & -1711.479296 & -1711.583030 \\
\hline 1-acetaldehyde, kinetic product & & -1557.244316 & -1556.632091 & -1556.722856 \\
\hline 1-acetaldehyde, thermodynamic product & & -1557.260234 & -1556.647961 & -1556.738012 \\
\hline $\mathbf{2} \cdot(\mathrm{EtOH})_{2}$ & & -1714.568183 & -1713.827420 & -1713.938491 \\
\hline TS2 & $i=-41.75$ & -1714.537761 & -1713.801378 & -1713.910035 \\
\hline Int 1 & & -1714.538091 & -1713.800874 & -1713.910587 \\
\hline TS3 & $i=-1584.19$ & -1714.534064 & -1713.800018 & -1713.910284 \\
\hline Int 2 & & -1714.550946 & -1713.811680 & -1713.923871 \\
\hline Int 3 & & -1713.353651 & -1712.634011 & -1712.745076 \\
\hline TS4 & $i=-59.80$ & -1713.353415 & -1712.634899 & -1712.743255 \\
\hline $4 \cdot \mathrm{EtOH}$ & & -1713.390461 & -1712.669056 & -1712.776860 \\
\hline TS5 & $i=-1463.28$ & -1713.349081 & -1712.633572 & -1712.744616 \\
\hline TS6 & $i=-1569.30$ & -1713.367611 & -1712.650587 & -1712.755751 \\
\hline Int 4 & & -1558.441782 & -1557.806580 & -1557.901793 \\
\hline Int 4-EtOH & & -1713.379249 & -1712.654986 & -1712.761296 \\
\hline TS7 & $i=-129.40$ & -1713.354278 & -1712.634464 & -1712.739270 \\
\hline Int 5 & & -1713.358715 & -1712.639055 & -1712.745089 \\
\hline TS8 & $i=-505.81$ & -1713.350376 & -1712.635107 & -1712.742819 \\
\hline TS9 & $i=-1658.74$ & -1713.348511 & -1712.632214 & -1712.737639 \\
\hline TS11 & $i=-99.19$ & -1712.147427 & -1711.451046 & -1711.553624 \\
\hline
\end{tabular}




\begin{tabular}{|l|r|l|l|l|}
\hline Int 7 & -1712.153529 & -1711.456888 & -1711.560348 \\
\hline TS13 & $I=-1571.62$ & -1558.423484 & -1557.794092 & -1557.887988 \\
\hline Int 8 & -1404.688802 & -1404.123438 & -1404.210321 \\
\hline
\end{tabular}

Table S2. MN15-L energies (in toluene solvent continuum).

\begin{tabular}{|c|c|c|c|c|}
\hline species & Imag. Freq. & $E$ & $\mathrm{H}$ & $G$ \\
\hline $\mathrm{H}_{2}$ (1 atm) & & -1.166536 & -1.148737 & -1.163570 \\
\hline Acetaldehyde & & -153.552297 & -153.492182 & -153.521954 \\
\hline $\mathrm{EtOH}$ & & -154.747061 & -154.661967 & -154.692590 \\
\hline EtOAc & & -307.151486 & -307.025273 & -307.066052 \\
\hline $\mathrm{H}_{2} \mathrm{O}$ & & -76.288282 & -76.262805 & -76.284897 \\
\hline 1-(ethoxy)ethanol & & -308.320067 & -308.170622 & -308.210964 \\
\hline 1 & & -1402.057901 & -1401.510616 & -1401.594406 \\
\hline $\mathbf{1} \cdot(\mathrm{EtOH})_{2}$ & & -1711.587329 & -1710.865006 & -1710.972750 \\
\hline 2 & & -1403.248780 & -1402.681883 & -1402.765944 \\
\hline $2^{\prime}$ & & -1403.241636 & -1402.674074 & -1402.758121 \\
\hline TS1 & $i=-1171.64$ & -1403.200046 & -1402.638299 & -1402.722231 \\
\hline Int 8 & & -1403.227399 & -1402.662237 & -1402.747356 \\
\hline Complex 4 & & -1556.834432 & -1556.200057 & -1556.292593 \\
\hline Complex 4' & & -1556.831890 & -1556.197316 & -1556.290040 \\
\hline $\mathrm{RuH}(\mathrm{OH})(\mathrm{CO})\left[\mathrm{Et}_{2} \mathrm{NCH}_{2} \mathrm{PyCH}_{2} \mathrm{PtBu}_{2}\right]$ & & -1478.367964 & -1477.792079 & -1477.879654 \\
\hline $\begin{array}{l}\text { 1-(ethoxy)ethoxide, } \\
\text { RuH }\left\{\mathrm{OCH}(\mathrm{OEt}) \mathrm{CH}_{3}\right\}(\mathrm{CO})\left[\mathrm{Et}_{2} \mathrm{NCH}_{2} \mathrm{PyCH}_{2} \mathrm{PtBu}_{2}\right]\end{array}$ & & -1710.407897 & -1709.708214 & -1709.808625 \\
\hline 1-acetaldehyde, kinetic product & & -1555.642751 & -1555.030376 & -1555.119418 \\
\hline 1-acetaldehyde, thermodynamic product & & -1555.659358 & -1555.047142 & -1555.135165 \\
\hline $\mathbf{2} \cdot(\mathrm{EtOH})_{2}$ & & -1712.781867 & -1712.040105 & -1712.147080 \\
\hline TS2 & $i=-379.08$ & -1712.747739 & -1712.010529 & -1712.114334 \\
\hline Int 1 & & -1712.748374 & -1712.009666 & -1712.113854 \\
\hline TS3 & $i=-753.73$ & -1712.742283 & -1712.008271 & -1712.114441 \\
\hline Int 2 & & -1712.762174 & -1712.021988 & -1712.130800 \\
\hline Int 3 & & -1711.573656 & -1710.852464 & -1710.956447 \\
\hline TS4 & $i=-87.12$ & -1711.573267 & -1710.852351 & -1710.954887 \\
\hline $4 \cdot \mathrm{EtOH}$ & & -1711.610311 & -1710.887851 & -1710.991298 \\
\hline TS5 & $i=-1008.71$ & -1711.563178 & -1710.846198 & -1710.950270 \\
\hline TS6 & $i=-1271.3$ & -1711.580652 & -1710.863182 & -1710.965318 \\
\hline Int 4 & & -1556.825463 & -1556.189668 & -1556.284040 \\
\hline Int 4.EtOH & & -1711.597417 & -1710.873667 & -1710.978964 \\
\hline Int 5 & & -1711.574339 & -1710.854951 & -1710.961765 \\
\hline
\end{tabular}




\section{Supporting Information}

\begin{tabular}{|l|l|l|l|l|}
\hline TS8 & $i=-626.22$ & -1711.569298 & -1710.853573 & -1710.959804 \\
\hline TS10 & $i=-137.12$ & -1710.389805 & -1709.690988 & -1709.788915 \\
\hline Int 6 & & -1710.389874 & -1709.689922 & -1709.788918 \\
\hline TS11 & $i=-84.73$ & -1710.375392 & -1709.678182 & -1709.777001 \\
\hline Int 7 & & -1710.379015 & -1709.681807 & -1709.782590 \\
\hline TS12 & $i=-693.91$ & -1710.375091 & -1709.681295 & -1709.780925 \\
\hline TS13 & $I=-1545.61$ & -1556.804557 & -1556.174554 & -1556.266108 \\
\hline Int 8 & & -1403.227399 & -1402.662237 & -1402.747356 \\
\hline
\end{tabular}

The basis sets used in the calciulations:

HCNOPO

def2SVP

****

$\mathrm{HO}$

P 111.00

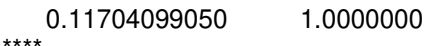

C 0

S 111.00

$0.67053540256 \mathrm{E}-01 \quad 1.0000000$

D 11.00

$0.11713185140 \quad 1.0000000$

$\mathrm{N} 0$

S 111.00

0.96171241529E-01 $\quad 1.0000000$

D 11.00

$0.16697708112 \quad 1.0000000$

O 0

S 111.00

$0.76572453250 \mathrm{E}-01 \quad 1.0000000$

P 11.00 $0.69002276350 \mathrm{E}-01 \quad 1.0000000$

D 11.00

$0.17992024323 \quad 1.0000000$

P 0

S 111.00

$0.64309734755 \mathrm{E}-01 \quad 1.0000000$

D 11.00

$0.99587311089 \mathrm{E}-01 \quad 1.0000000$

Ru 0

Def2QZVP

****

Ru 0

def2 


\section{X-ray Structure Report}

\section{Introduction}

Dr. Paul D. Boyle, Department of Chemistry X-Ray Facility, Western University, is acknowledged for assistance with the X-ray data collection.

$\mathrm{H}$-atoms, except $\mathrm{H} 1$ and $\mathrm{H} 2$, were introduced in calculated positions and refined on a riding model. All other atoms (including $\mathrm{H} 1$ and $\mathrm{H} 2$ ) were introduced in difference map positions and refined isotropically ( $\mathrm{H} 1$ and $\mathrm{H} 2$ ) or anisotropically (all non-hydrogen atoms.)

A numbering scheme consistent with a previously reported structure was used (except for calculated hydrogens, where the numbering scheme in the current model depends on the parent atom):

E.Balaraman, C.Gunanathan, Jing Zhang, L.J.W.Shimon, D.Milstein Nature Chemistry (2011), 3, 609. (CCDC 826775 and Refcode EYEPOO)
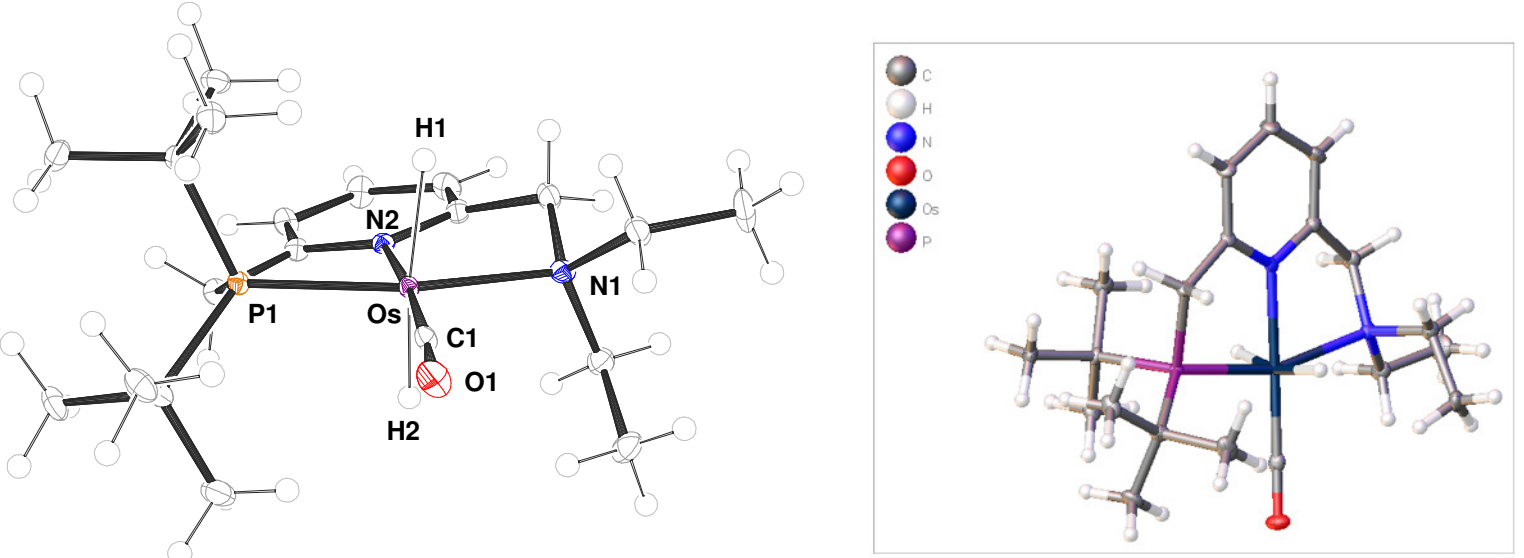

\section{Experimental}

A single crystal of $\mathrm{C}_{20} \mathrm{H}_{37} \mathrm{~N}_{2} \mathrm{OOSP}$ was selected and collected on a Bruker APEX-II CCD diffractometer. The crystal was kept at 110(2) K during data collection. Using Olex2 [1], the structure was solved with the ShelXT [2] structure solution program using Direct Methods and refined with the ShelXL [3] refinement package using Least Squares minimisation.

1. Dolomanov, O.V., Bourhis, L.J., Gildea, R.J, Howard, J.A.K. \& Puschmann, H. (2009), J. Appl. Cryst. 42, 339-341.

2. Sheldrick, G.M. (2015). Acta Cryst. A71, 3-8.

3. Sheldrick, G.M. (2015). Acta Cryst. C71, 3-8.

\section{Crystal structure determination}

Crystal Data for $\mathrm{C}_{20} \mathrm{H}_{37} \mathrm{~N}_{2} \mathrm{OOsP}(M=542.68 \mathrm{~g} / \mathrm{mol})$ : monoclinic, space group $\mathrm{P} 2{ }_{1} / \mathrm{n}$ (no. 14), $a=12.7004(3) \AA, b=13.9199(3) \AA, c=12.7442(3) \AA, \beta=108.478(3)^{\circ}, V=2136.87(9) \AA^{3}, Z=4, T=$ $110(2) \mathrm{K}, \mu(\mathrm{MoK} \alpha)=6.053 \mathrm{~mm}^{-1}$, Dcalc $=1.687 \mathrm{~g} / \mathrm{cm}^{3}, 37214$ reflections measured $\left(4.464^{\circ} \leq 2 \theta \leq\right.$ $\left.52.744^{\circ}\right), 4381$ unique (4345 with $\left.\mathrm{I}>2 \sigma(\mathrm{I}) ; R_{\text {int }}=0.0546, \mathrm{R}_{\text {sigma }}=0.0225\right)$ which were used in all calculations. The final $R_{1}$ was 0.0342 (I $\left.>2 \sigma(\mathrm{I})\right)$ and $w R_{2}$ was 0.0712 (all data). 
Table S3 Crystal data and structure refinement for b19141.

\begin{tabular}{|c|c|}
\hline Identification code & DG-1 (Western b19141) \\
\hline Empirical formula & $\mathrm{C}_{20} \mathrm{H}_{37} \mathrm{~N}_{2} \mathrm{OOsP}$ \\
\hline Formula weight & 542.68 \\
\hline Temperature/K & $110(2)$ \\
\hline Crystal system & monoclinic \\
\hline Space group & $\mathrm{P} 2{ }_{1} / \mathrm{n}$ \\
\hline $\mathrm{a} / \AA$ & $12.7004(3)$ \\
\hline $\mathrm{b} / \AA$ & $13.9199(3)$ \\
\hline $\mathrm{c} / \AA$ & $12.7442(3)$ \\
\hline$\alpha /^{\circ}$ & 90 \\
\hline$\beta /^{\circ}$ & $108.478(3)$ \\
\hline$\gamma /{ }^{\circ}$ & 90 \\
\hline Volume $/ \AA^{3}$ & 2136.87(9) \\
\hline $\mathrm{Z}$ & 4 \\
\hline$\rho_{\text {calc }} \mathrm{g} / \mathrm{cm}^{3}$ & 1.687 \\
\hline$\mu / \mathrm{mm}^{-1}$ & 6.053 \\
\hline $\mathrm{F}(000)$ & 1080.0 \\
\hline Crystal size $/ \mathrm{mm}^{3}$ & $0.120 \times 0.102 \times 0.045$ \\
\hline Radiation & $\operatorname{MoK} \alpha(\lambda=0.71073)$ \\
\hline \multicolumn{2}{|c|}{$2 \Theta$ range for data collection $/{ }^{\circ} 4.464$ to 52.744} \\
\hline Index ranges & $-15 \leq \mathrm{h} \leq 15,-16 \leq \mathrm{k} \leq 17,-15 \leq 1 \leq 15$ \\
\hline Reflections collected & 37214 \\
\hline Independent reflections & $\begin{array}{l}4381[4345 \text { with } \mathrm{I}>2 \sigma(\mathrm{I}) \\
\left.\mathrm{R}_{\text {int }}=0.0546, \mathrm{R}_{\text {sigma }}=0.0225\right]\end{array}$ \\
\hline Data/restraints/parameters & $4381 / 0 / 242$ \\
\hline Goodness-of-fit on $\mathrm{F}^{2}$ & 1.309 \\
\hline Final $R$ indexes $[\mathrm{I}>=2 \sigma(\mathrm{I})]$ & $\mathrm{R}_{1}=0.0342, \mathrm{wR}_{2}=0.0710$ \\
\hline Final $\mathrm{R}$ indexes [all data] & $\mathrm{R}_{1}=0.0347, \mathrm{wR}_{2}=0.0712$ \\
\hline Largest diff. peak/hole / e $\AA$ & $2.06 /-1.96$ \\
\hline
\end{tabular}


Table S4 Fractional Atomic Coordinates $\left(\times 10^{4}\right)$ and Equivalent Isotropic Displacement Parameters $\left(\AA^{2} \times 10^{3}\right)$ for b19141. $U_{\text {eq }}$ is defined as $1 / 3$ of of the trace of the orthogonalised $U_{I J}$ tensor.

\begin{tabular}{lrrrr} 
Atom & \multicolumn{1}{l}{$\boldsymbol{x}$} & \multicolumn{1}{l}{$\boldsymbol{z}$} & \multicolumn{1}{l}{$\mathbf{U}(\mathbf{e q})$} \\
Os1 & $5368.2(2)$ & $2604.0(2)$ & $3686.6(2)$ & $8.22(7)$ \\
P1 & $5353.6(9)$ & $2853.8(8)$ & $1939.2(9)$ & $8.8(2)$ \\
O1 & $7081(3)$ & $1035(3)$ & $4106(3)$ & $17.9(7)$ \\
N1 & $4937(3)$ & $2756(3)$ & $5250(3)$ & $11.2(8)$ \\
N2 & $4072(3)$ & $3619(3)$ & $3240(3)$ & $9.8(8)$ \\
C1 & $6441(4)$ & $1664(3)$ & $3976(4)$ & $12.5(9)$ \\
C2 & $3566(4)$ & $3865(3)$ & $2177(4)$ & $10.5(9)$ \\
C3 & $2714(4)$ & $4544(4)$ & $1906(4)$ & $14.1(10)$ \\
C4 & $2378(4)$ & $4942(4)$ & $2742(4)$ & $16.5(10)$ \\
C5 & $2899(4)$ & $4671(4)$ & $3839(4)$ & $16.9(10)$ \\
C6 & $3759(4)$ & $4014(3)$ & $4060(4)$ & $12.0(9)$ \\
C7 & $4497(4)$ & $3755(3)$ & $5196(4)$ & $14.8(10)$ \\
C8 & $3938(4)$ & $3351(3)$ & $1326(4)$ & $11.9(9)$ \\
C9 & $4067(4)$ & $2083(3)$ & $5355(4)$ & $14.7(10)$ \\
C10 & $5974(4)$ & $2678(4)$ & $6240(4)$ & $15.2(10)$ \\
C11 & $4436(4)$ & $1036(4)$ & $5495(4)$ & $18.6(11)$ \\
C12 & $5823(5)$ & $2829(4)$ & $7372(4)$ & $25.5(12)$ \\
C13 & $5360(4)$ & $1819(3)$ & $982(4)$ & $13.7(10)$ \\
C14 & $6278(4)$ & $3847(3)$ & $1717(4)$ & $10.5(9)$ \\
C15 & $4798(4)$ & $2045(4)$ & $-247(4)$ & $17.6(10)$ \\
C16 & $6560(4)$ & $1483(4)$ & $1172(4)$ & $16.9(10)$ \\
C17 & $4718(5)$ & $982(4)$ & $1274(5)$ & $20.6(11)$ \\
C18 & $6236(4)$ & $4013(4)$ & $517(4)$ & $16.3(10)$ \\
C19 & $5895(4)$ & $4784(3)$ & $2137(4)$ & $15.4(10)$ \\
C20 & $7480(4)$ & $3663(4)$ & $2442(4)$ & $15.8(10)$
\end{tabular}


Table S5 Anisotropic Displacement Parameters $\left(\AA^{2} \times 10^{3}\right)$ for b19141. The Anisotropic displacement factor exponent takes the form: $-2 \pi^{2}\left[h^{2} a^{* 2} U_{11}+2 h k a * b * U_{12}+\ldots\right]$.

\begin{tabular}{lrrrrrr} 
Atom & \multicolumn{1}{c}{$\mathbf{U 1 1}_{11}$} & \multicolumn{1}{c}{$\mathbf{U}_{22}$} & \multicolumn{1}{c}{$\mathbf{U}_{\mathbf{3 3}}$} & \multicolumn{1}{c}{$\mathbf{U}_{13}$} & \multicolumn{1}{c}{$\mathbf{U}_{\mathbf{1 2}}$} \\
Os1 & $8.26(10)$ & $8.64(10)$ & $7.13(10)$ & $0.10(6)$ & $1.54(6)$ & $0.70(6)$ \\
P1 & $7.3(5)$ & $10.3(5)$ & $7.9(5)$ & $0.3(4)$ & $1.0(4)$ & $0.2(4)$ \\
O1 & $18.1(18)$ & $17.2(18)$ & $15.5(18)$ & $-0.9(14)$ & $1.1(14)$ & $7.3(14)$ \\
N1 & $8.3(18)$ & $12.1(19)$ & $11.8(19)$ & $0.0(15)$ & $1.1(15)$ & $-1.3(15)$ \\
N2 & $8.2(18)$ & $10.4(18)$ & $11.2(19)$ & $-1.3(15)$ & $3.9(15)$ & $-2.2(14)$ \\
C1 & $14(2)$ & $15(2)$ & $7(2)$ & $-1.6(17)$ & $1.2(18)$ & $-3.9(18)$ \\
C2 & $6(2)$ & $14(2)$ & $11(2)$ & $-0.1(17)$ & $1.1(17)$ & $-1.1(17)$ \\
C3 & $8(2)$ & $19(2)$ & $11(2)$ & $1.1(19)$ & $-1.7(18)$ & $2.3(18)$ \\
C4 & $11(2)$ & $21(3)$ & $18(3)$ & $3(2)$ & $3.9(19)$ & $6.3(19)$ \\
C5 & $17(2)$ & $17(2)$ & $20(3)$ & $-5(2)$ & $10(2)$ & $2.3(19)$ \\
C6 & $11(2)$ & $13(2)$ & $12(2)$ & $0.0(18)$ & $3.7(18)$ & $-0.6(17)$ \\
C7 & $19(3)$ & $15(2)$ & $11(2)$ & $-1.8(18)$ & $5(2)$ & $0.8(19)$ \\
C8 & $8(2)$ & $14(2)$ & $11(2)$ & $1.4(18)$ & $-0.6(18)$ & $1.6(17)$ \\
C9 & $15(2)$ & $15(2)$ & $15(2)$ & $0.7(19)$ & $4.8(19)$ & $-0.7(19)$ \\
C10 & $17(2)$ & $16(2)$ & $11(2)$ & $-1.0(18)$ & $2.4(19)$ & $0.3(19)$ \\
C11 & $22(3)$ & $17(2)$ & $21(3)$ & $3(2)$ & $13(2)$ & $-1(2)$ \\
C12 & $34(3)$ & $33(3)$ & $8(2)$ & $1(2)$ & $5(2)$ & $5(2)$ \\
C13 & $12(2)$ & $14(2)$ & $14(2)$ & $-4.1(18)$ & $3.0(19)$ & $-0.1(18)$ \\
C14 & $12(2)$ & $12(2)$ & $8(2)$ & $1.3(17)$ & $2.2(18)$ & $-2.4(17)$ \\
C15 & $16(2)$ & $27(3)$ & $10(2)$ & $-6(2)$ & $2.6(19)$ & $-3(2)$ \\
C16 & $16(2)$ & $19(2)$ & $17(3)$ & $-7(2)$ & $7(2)$ & $2.3(19)$ \\
C17 & $25(3)$ & $17(3)$ & $23(3)$ & $-9(2)$ & $12(2)$ & $-7(2)$ \\
C18 & $19(3)$ & $18(2)$ & $14(2)$ & $3.2(19)$ & $7(2)$ & $-2.0(19)$ \\
C19 & $19(3)$ & $11(2)$ & $15(2)$ & $-1.5(18)$ & $3(2)$ & $-2.2(18)$ \\
C20 & $10(2)$ & $23(3)$ & $13(2)$ & $-0.9(19)$ & $2.3(19)$ & $-3.6(19)$
\end{tabular}


Table S6 Bond Lengths for b19141.

\section{Atom Atom Length/Å}

Os1 P1 2.2482(12)

Os1 N1 2.238(4)

Os1 N2 $2.106(4)$

Os1 C1 $1.840(5)$

$\mathrm{P} 1 \mathrm{C} 8 \quad 1.853(5)$

P1 C13 1.889(5)

$\mathrm{P} 1 \mathrm{C} 14 \quad 1.893(5)$

O1 C1 $1.171(6)$

N1 C7 $1.493(6)$

N1 C9 $1.486(6)$

N1 C10 $1.511(6)$

N2 C2 $1.347(6)$

N2 C6 $1.347(6)$

C2 C3 1.395(6)

\section{Atom Atom Length/Å}

C2 C8

$1.495(7)$

C3 C4 1.384(7)

C4 C5 $1.395(7)$

C5 C6 $1.383(7)$

C6 C7 1.498(7)

C9 C11 $1.525(7)$

C10 C12 1.529(7)

C13 C15 1.534(7)

C13 C16 1.539(7)

C13 C17 1.533(7)

C14 C18 $1.530(6)$

C14 C19 1.545(7)

C14 C20 1.535 (6) 
Table S7 Bond Angles for b19141.

\begin{tabular}{lllrllll} 
Atom & Atom Atom & \multicolumn{1}{c}{ Angle $^{\circ}$} & & \multicolumn{2}{c}{ Atom Atom Atom } & Angle $^{\circ}$ \\
N1 & Os1 & P1 & $160.05(11)$ & C3 & C2 & C8 & $122.9(4)$ \\
N2 & Os1 & P1 & $82.44(11)$ & C4 & C3 & C2 & $119.0(4)$ \\
N2 & Os1 & N1 & $77.65(15)$ & C3 & C4 & C5 & $120.0(4)$ \\
C1 & Os1 & P1 & $94.69(15)$ & C6 & C5 & C4 & $118.4(5)$ \\
C1 & Os1 & N1 & $105.26(18)$ & N2 & C6 & C5 & $121.3(4)$ \\
C1 & Os1 & N2 & $175.46(17)$ & N2 & C6 & C7 & $113.8(4)$ \\
C8 & P1 & Os1 & $100.24(16)$ & C5 & C6 & C7 & $124.6(4)$ \\
C8 & P1 & C13 & $102.4(2)$ & N1 & C7 & C6 & $112.3(4)$ \\
C8 & P1 & C14 & $103.2(2)$ & C2 & C8 & P1 & $111.1(3)$ \\
C13 & P1 & Os1 & $121.40(16)$ & N1 & C9 & C11 & $113.8(4)$ \\
C13 & P1 & C14 & $109.3(2)$ & N1 & C10 & C12 & $116.2(4)$ \\
C14 & P1 & Os1 & $116.79(15)$ & C15 & C13 & P1 & $114.0(3)$ \\
C7 & N1 & Os1 & $103.9(3)$ & C15 & C13 & C16 & $109.9(4)$ \\
C7 & N1 & C10 & $108.6(4)$ & C16 & C13 & P1 & $109.7(3)$ \\
C9 & N1 & Os1 & $114.8(3)$ & C17 & C13 & P1 & $108.1(3)$ \\
C9 & N1 & C7 & $108.3(4)$ & C17 & C13 & C15 & $107.0(4)$ \\
C9 & N1 & C10 & $110.8(4)$ & C17 & C13 & C16 & $107.9(4)$ \\
C10 & N1 & Os1 & $110.0(3)$ & C18 & C14 & P1 & $115.7(3)$ \\
C2 & N2 & Os1 & $121.7(3)$ & C18 & C14 & C19 & $107.7(4)$ \\
C6 & N2 & Os1 & $117.5(3)$ & C18 & C14 & C20 & $109.9(4)$ \\
C6 & N2 & C2 & $120.8(4)$ & C19 & C14 & P1 & $106.6(3)$ \\
O1 & C1 & Os1 & $175.8(4)$ & C20 & C14 & P1 & $109.5(3)$ \\
N2 & C2 & C3 & $120.4(4)$ & C20 & C14 & C19 & $107.1(4)$ \\
N2 & C2 & C8 & $116.7(4)$ & & & & \\
& & & & & & &
\end{tabular}


Table S8 Hydrogen Atom Coordinates $\left(\AA \times 10^{4}\right)$ and Isotropic Displacement Parameters $\left(\AA^{2} \times 10^{3}\right)$ for b19141.

\begin{tabular}{|c|c|c|c|c|}
\hline Atom & $x$ & $y$ & $z$ & $\mathbf{U}(\mathbf{e q})$ \\
\hline $\mathrm{H} 1$ & $4330(50)$ & $1850(50)$ & $3300(50)$ & $29(17)$ \\
\hline $\mathrm{H} 2$ & $6260(50)$ & $3540(50)$ & $4230(50)$ & $32(18)$ \\
\hline H3 & 2369.7 & 4729.99 & 1157.71 & 17 \\
\hline $\mathrm{H} 4$ & 1793.16 & 5399.22 & 2570.37 & 20 \\
\hline H5 & 2669.2 & 4932.45 & 4419.4 & 20 \\
\hline H7A & 5127.06 & 4211.47 & 5423.32 & 18 \\
\hline H7B & 4073.82 & 3818.97 & 5725.07 & 18 \\
\hline H8A & 3931.54 & 3802.32 & 723.69 & 14 \\
\hline H8B & 3413.93 & 2822.62 & 1002.74 & 14 \\
\hline H9A & 3407.84 & 2140.42 & 4687.27 & 18 \\
\hline H9B & 3841.44 & 2277.05 & 6000.27 & 18 \\
\hline $\mathrm{H} 10 \mathrm{~A}$ & 6517.63 & 3156.06 & 6153.87 & 18 \\
\hline H10B & 6300.22 & 2033.45 & 6231.69 & 18 \\
\hline H11A & 4697.83 & 846.07 & 4880.08 & 28 \\
\hline H11B & 3808.22 & 628.6 & 5501 & 28 \\
\hline $\mathrm{H} 11 \mathrm{C}$ & 5039.04 & 958.94 & 6195.19 & 28 \\
\hline $\mathrm{H} 12 \mathrm{~A}$ & 5532.74 & 3475.84 & 7409.32 & 38 \\
\hline H12B & 6540.87 & 2755.87 & 7952.43 & 38 \\
\hline $\mathrm{H} 12 \mathrm{C}$ & 5299.95 & 2351.77 & 7480.75 & 38 \\
\hline $\mathrm{H} 15 \mathrm{~A}$ & 4039.69 & 2271.62 & -359.49 & 26 \\
\hline H15B & 4775.53 & 1462.52 & -685.76 & 26 \\
\hline $\mathrm{H} 15 \mathrm{C}$ & 5220.03 & 2544.29 & -480.15 & 26 \\
\hline H16A & 6962.81 & 1975.71 & 902.97 & 25 \\
\hline H16B & 6553.7 & 881.91 & 769.74 & 25 \\
\hline H16C & 6928.88 & 1377.71 & 1964.27 & 25 \\
\hline H17A & 5047.99 & 820.51 & 2058.5 & 31 \\
\hline H17B & 4753.67 & 422.28 & 820.86 & 31 \\
\hline $\mathrm{H} 17 \mathrm{C}$ & 3940.74 & 1170.03 & 1129.27 & 31 \\
\hline H18A & 6538.66 & 3449.69 & 250.36 & 24 \\
\hline H18B & 6678.04 & 4580.6 & 477.95 & 24 \\
\hline $\mathrm{H} 18 \mathrm{C}$ & 5464.76 & 4113.57 & 55.26 & 24 \\
\hline H19A & 5133.08 & 4932.61 & 1681.36 & 23 \\
\hline H19B & 6387.19 & 5311.96 & 2086.63 & 23 \\
\hline H19C & 5922.5 & 4700.67 & 2908.43 & 23 \\
\hline $\mathrm{H} 20 \mathrm{~A}$ & 7510.86 & 3615.13 & 3218.24 & 24 \\
\hline H20B & 7951.78 & 4194.93 & 2356.44 & 24 \\
\hline $\mathrm{H} 20 \mathrm{C}$ & 7744.48 & 3061.54 & 2213.84 & 24 \\
\hline
\end{tabular}

